\title{
Radiomics in Stroke Neuroimaging: Techniques, Applications, and Challenges
}

\author{
Qian Chen, Tianyi Xia, Mingyue Zhang, Nengzhi Xia, Jinjin Liu, Yunjun Yang*
}

Department of Radiology, The First Affiliated Hospital of Wenzhou Medical University, Zhejiang, China.

[Received March 21, 2020; Revised April 21, 2020; Accepted April 21, 2020]

\begin{abstract}
Stroke is a leading cause of disability and mortality worldwide, resulting in substantial economic costs for post-stroke care each year. Neuroimaging, such as cranial computed tomography or magnetic resonance imaging, is the backbone of stroke management strategies, which can guide treatment decision-making (thrombolysis or hemostasis) at an early stage. With advances in computational technologies, particularly in machine learning, visual image information can now be converted into numerous quantitative features in an objective, repeatable, and high-throughput manner, in a process known as radiomics. Radiomics is mainly used in the field of oncology, which remains an area of active research. Over the past few years, investigators have attempted to apply radiomics to stroke in the hope of gaining benefits similar to those obtained in cancer management, i.e., in promoting the development of personalized precision medicine. Currently, radiomic analysis has shown promise for a variety of applications in stroke, including the diagnosis of stroke lesions, early prediction of outcomes, and evaluation for long-term prognosis. In this article, we elaborate the contributions of radiomics to stroke, as well as the subprocesses and techniques involved in radiomics studies. We also discuss the potential challenges facing its widespread implementation in routine practice and the directions for future research.
\end{abstract}

Key words: decision-making, neuroimaging, radiomics, stroke, texture analysis

Stroke is a vascular event characterized by a sudden onset of focal neurologic deficits in the relevant part of the central nervous system, including ischemic stroke, intracerebral hemorrhage (ICH), and subarachnoid hemorrhage (SAH) [1]. Globally, it represents a leading cause of mortality and disability, and there were an estimated 80 million stroke survivors in 2016, resulting in substantial economic costs for post-stroke care [2, 3]. Despite the declining age-standardized mortality rates over the past 2 decades, the absolute number of incident stroke, disability-adjusted life-years lost due to stroke, and stroke-related deaths is increasing [3]. Along with population growth and aging, the burden of stroke is likely to increase further.

Medical imaging, such as X-ray computed tomography $(\mathrm{CT})$ or magnetic resonance imaging (MRI), promotes the development of personalized diagnosis and treatment in cancer, by complementing with genomic, proteomic, and metabolomic technologies [4, 5]. Neuroimaging as a part of imaging protocols, plays a vital role in stroke analytics in both clinical practice and trials. In acute ischemic stroke (AIS), the results from recent clinical trials with imaging features as criteria for selection of subjects for therapy confirmed the improved efficacy of endovascular thrombectomy compared to standard medical care with intravenous alteplase [6, 7]. Additionally, the perfusion-diffusion mismatch determined by advanced neuroimaging has shown good potential for delayed interventions, wherein thrombolytic treatment for target-penumbra can be undertaken beyond the $4.5 \mathrm{~h}$ time point [8]. In acute ICH, some randomized clinical trials (e.g., ATACH, INTERACT, or SPOT-

*Correspondence should be addressed to: Dr. Yunjun Yang. Department of Radiology, The First Affiliated Hospital of Wenzhou Medical University, Wenzhou 325000, Zhejiang, China. Email: yyjunjim@163.com.

Copyright: ( $) 2020$ Chen Q et al. This is an open-access article distributed under the terms of the Creative Commons Attribution License, which permits unrestricted use, distribution, and reproduction in any medium, provided the original author and source are credited. 
AUST) have been performed, with the goal of improving prognosis for patients with a high risk of early hematoma growth using baseline neuroimaging markers [9-12].

During the past decade, advances in computational technologies, particularly in machine learning, have placed medical imaging in an increasingly central role in patient-specific management. This progress makes it possible to convert subjective visual interpretation into an objective assessment that is driven by image data. Radiomics has emerged in this context. It is a computeraided process, in which a profuse number of quantitative features (e.g., shape, intensity or texture) can be extracted from biomedical images in an objective, reproducible, and high-throughput manner [13-15]. This process is motivated by the concept that digitally encrypted images contain biologic information related to the pathophysiology of certain diseases, and this information can be exploited via quantitative image analyses [16]. In the oncology field, the potential of radiomics arises from its ability to allow quantitative assessment of intratumor heterogeneity that reflects phenotype and/or microenvironment, which may not be visually perceived $[17,18]$; radiomic data, in combination with other medical information such as demographic, clinical, histologic or genomic data, can be used for clinical-decision support systems to improve treatment decision-making and accelerate advancements toward precision medicine in cancer $[5,16,19,20]$.
Radiomics has also shown promise for a variety of applications in stroke, facilitating its personalized management at an early stage. In this review, we describe the workflow of radiomics as well as its basic techniques. We also outline the main contributions of radiomics to stroke, with an emphasis on the diagnostic, predictive, and prognostic value of radiomics during post-stroke care. Finally, we discuss the potential challenges facing the widespread implementation of radiomics in routine clinical practice and the directions for future research.

\section{Radiomic workflow and techniques}

Radiomics is a high-throughput process, during which a quantitative relationship can be established between multimode data sources by converting medical images into numerous radiomic features. This quantitative relationship is expected to address a clinically relevant problem in certain diseases, such as early diagnosis or accurate prognosis prediction, thereby improving treatment decisions. Regardless of the lesion type and clinical purpose, the workflow of radiomics is similar. Radiomic analysis involves a spectrum of continuous subprocesses, including image data acquisition, segmentation, feature extraction, exploratory analysis, and modeling (Fig. 1).

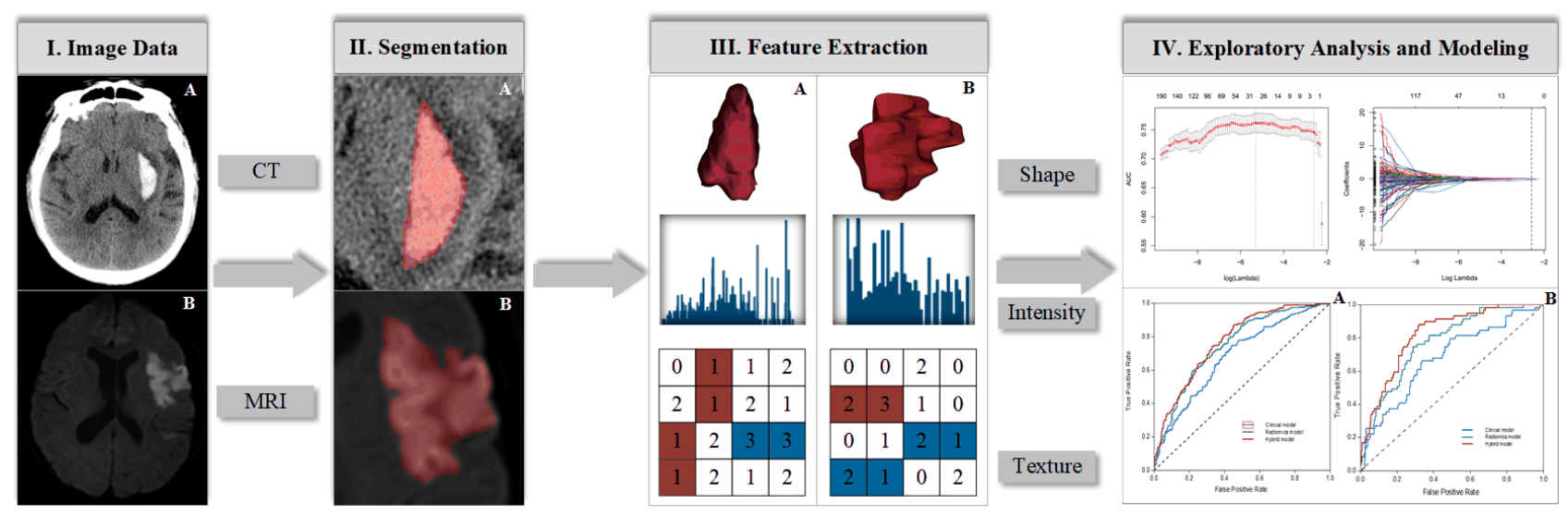

Figure 1. Flowchart shows the typical process of radiomics in stroke neuroimaging. (A) Example CT image of an intracerebral hemorrhage and (B) example MR image of a patient with ischemic stroke.

\subsection{Image data acquisition}

The process of radiomics begins with digital imaging. Radiomic analyses can be applied to all imaging modalities (i.e., CT, MRI, PET, and ultrasound), but CT is the most commonly used technique. This is particularly useful in stroke, as non-contrast CT (NCCT) can rapidly determine the presence of an intracranial hemorrhage
[21]. Sufficient imaging data sources are beneficial to statistical inference, but heterogeneity in imaging protocols may cause unexpected effects on both the quality of the extracted features and the radiomic models $[22,23]$. For CT images, several studies have recently shown that the majorities of radiomic features are highly affected by image acquisition and reconstruction parameters and thus may be nonreproducible [24-26]. 
Results from a phantom study have shown that diverse scanners made by different manufacturers could cause variability in radiomic feature values [27]. Additionally, slice thicknesses as well as bin width of gray-level discretization also cause such variation [28].

Efforts are ongoing to enhance the stability of radiomic features via preprocessing techniques, such as normalization of the gray-level and voxel size or resampling of the pixel size to minimize feature dependency on voxel size [29, 30]. Nevertheless, progress has been limited to certain diseases or imaging modalities of the disease. To date, there is still a lack of evidence regarding the stability and reproducibility of radiomic features obtained from $\mathrm{CT}$ in either ischemic or hemorrhagic stroke. It should be noted that the robustness of radiomic features and comparability between radiomics studies can only be achieved through extensive disclosure of standardized imaging protocols [20].

\subsection{Segmentation}

Segmentation is the act that isolates a lesion of interest from the surrounding normal tissue. This represents the most critical step during radiomic workflow because it determines which region within an image is to be analyzed further and from where the radiomic feature is generated. This step involves variations in the selection of regions of interest (ROIs) and segmentation techniques.

Generally, the segmentation of ROIs is performed in 3 dimensions (3D) - that is, the entire lesion is extracted and analyzed, although $2 \mathrm{D}$ analysis at a single slice can also be carried out [31]. It is unknown how much lesion information should be harnessed to conclude robust results in radiomic analysis. Intuitively, 3D-radiomics could provide more information, particularly for lesions that are spatially heterogeneous and large in volume. However, volumetric assessment is time- and laborintensive, and more importantly, increases the risk of radiomic feature instability due to segmentation of ROIs from multiple image slices [32]. Although 3D-radiomics appears to be more valuable for evaluating tumor heterogeneity in patients with colorectal cancer [33], in those with other disorders, including stroke, there is little evidence to indicate that 3D-ROI outperforms 2D-ROI. Considering the pros and cons of both schemes, more attention should be paid to efficiency during the process of segmentation.
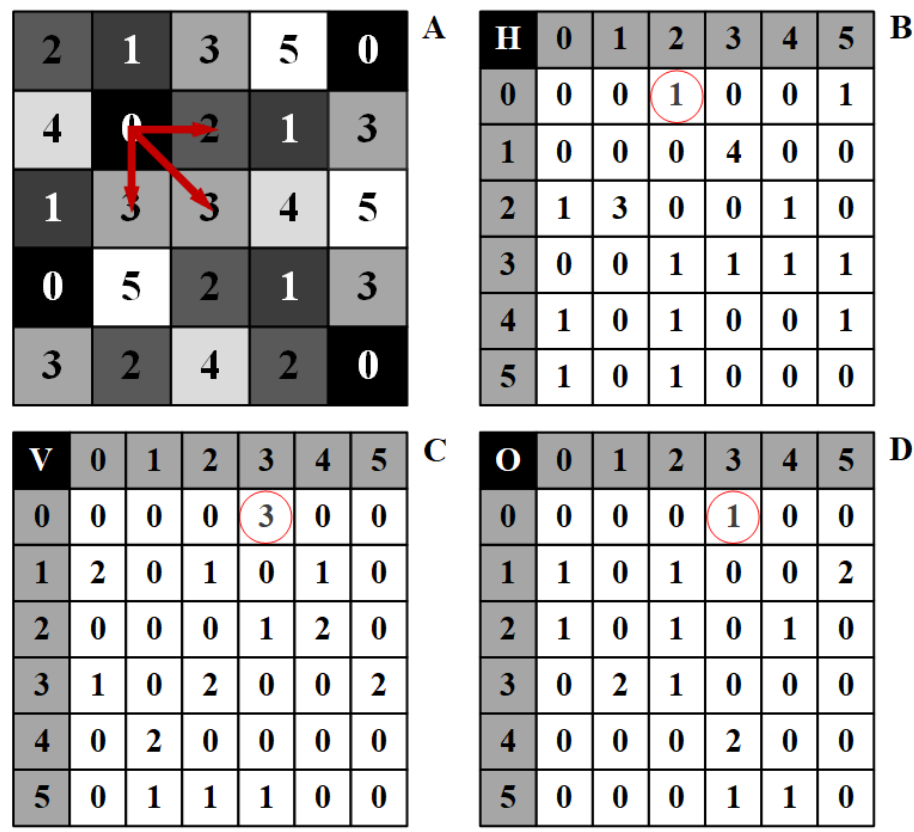

\begin{tabular}{|l|l|l|l|l|l|l|}
\hline O & 0 & 1 & 2 & 3 & 4 & 5 \\
\hline 0 & 0 & 0 & 0 & 1 & 0 & 0 \\
\hline 1 & 1 & 0 & 1 & 0 & 0 & 2 \\
\hline 2 & 1 & 0 & 1 & 0 & 1 & 0 \\
\hline 3 & 0 & 2 & 1 & 0 & 0 & 0 \\
\hline 4 & 0 & 0 & 0 & 2 & 0 & 0 \\
\hline 5 & 0 & 0 & 0 & 1 & 1 & 0 \\
\hline
\end{tabular}

Figure 2. Texture in radiomics. (A) A stylized graylevel image ( 5 x 5 pixels) with grey values ranging from 0 (black) to 5 (white) and its derived gray-level co-occurrence matrix (GLCM) in horizontal (B), vertical (C), and oblique (D) directions. Row and column numbers in the GLCM represent corresponding gray values, while cells in white contain the number of times corresponding gray values occurs adjacent to each other in three directions. For example, the frequency of gray values of 0,2 , and 3 (red arrows on the gray-level image) is then mapped onto corresponding cell of GLCM (red circles) each time they occur adjacent to each other in particular direction. In texture analysis, GLCM represents spatial interrelationship between pixels within a digital image.
Segmentation techniques include manual, semiautomatic, and fully automatic methods. Manual segmentation is time-consuming, which requires a trained observer to minimize the impact of inter-operator variability. In some cases, manual segmentation can be applied multiple times to ensure accuracy and reproducibility, particularly if the border of the lesion is unclear or if multiple focal lesions are observed at the same slice. Semi- or fully automatic segmentation is appealing, since each represents a more reproducible and faster way to enable observation of robust features from a given ROI. The Ischemic Stroke Lesion Segmentation (ISLES) challenge, a public competition that aims to explore the optimum segmentation method for stroke lesions, to some extent, promotes the development of tools for automation (www.isles-challenge.org/). 
Segmentation of the infarction core or peripheral penumbra on MR images can now be performed in a semior fully automated fashion in the context of machine learning [34-37]. For an ICH on CT images, automated segmentation algorithms based on random forest and open-source software (i.e., 3D-Slicer) have shown improved accuracy for determining hematoma volume over that of the conventional $\mathrm{ABC} / 2$ formula $[38,39]$. Currently, the addition of manual curation to computeraided edge detection is considered an ideal segmentation method with high reproducibility [16].

\subsection{Feature extraction}

Vast arrays of quantitative features can be extracted from multimodal images, which are broadly categorized as semantic metrics and agnostic metrics $[16,40]$. Semantic metrics refer to a set of features that can be captured by the naked eye and are commonly used in the radiology lexicon to describe the lesion's appearance characteristics. For example, "round", "oval", and "lobulated" are semantic descriptors of shape, which can help radiologists determine the irregularity of the lesion. Other semantic descriptors include size, location, and vascularity.

Agnostic metrics are calculated by mathematical algorithms and constitute the main body of radiomic features. Unlike semantic features, they cannot be obtained through visual interpretation. Agnostic metrics typically comprise first-, second-, and high-order statistical measures. First-order statistical measures describe the distribution of gray-level frequency from the pixel intensity histogram in a given ROI, without accounting for spatial interrelationships between pixels (e.g., maximum, median, skewness, and uniformity). Second-order statistical measures, that is, texture features, take into account both pixel intensity and statistical interrelationship in space (distance or orientation). They are the most widely used features in radiomics (Fig. 2) because texture analysis readily allows quantification of the heterogeneity within a lesion [32, 41, 42]. Texture features include co-occurrence matrix-based entropy, homogeneity, dissimilarity, correlation, etc. High-order statistical descriptors, such as busyness, contrast and coarseness, are calculated on matrices that consider relationships between 3 or more pixels.

In addition, radiomic analysis also employs a series of high-order statistical methods based on image filtration. Wavelet-transformed and Laplacian of Gaussian bandpass filters are the 2 most commonly used techniques [42, 43]. The former approach can convert spatial information into frequency and/or scale information in the direction of linear or radial waves (wavelet features) [44], while the latter allows extraction of texture features with different coarseness levels from a prescribed ROI while denoising and enhancing the edge of the images [45]. Several radiomics studies on neuroimages have used these filtration techniques to distinguish stable hematomas from those prone to enlargement [46-49].

\subsection{Exploratory analysis and modeling}

By making full use of various extraction techniques, the quantity of radiomic features is endless, in theory. However, the increased risk of redundancy among features may lead to overfitting, reducing the generalization and robustness of the model. Hence, an exploratory analysis should be performed before modeling.

\section{Feature selection}

The core work in exploratory analysis is feature selection. For the internal feature-selection method, clustering analysis can be used as a first step. This method enables highly correlated radiomic features to be simplified into archetypal features per cluster, thus revealing the general distributive tendencies of the data. When multiple sets of data are available (two or more), the intraclass correlation coefficient (ICC) is usually used to detect stable features (ICC > 0.8) and exclude those that are susceptible to the variation of ROI segmentation [17]. In the externalselection process, radiomic data should be analyzed along with other pertinent clinical data in a prediction model with a well-defined endpoint. Radiomic features that are highly correlated with clinical features cannot provide additional information and should be excluded before modeling. According to the clinical endpoint, univariate filters such as Student's t test or Mann-Whitney test, can sort features by quality [50]. The least absolute shrinkage and selection operator (LASSO) is a method commonly used for dimensionality reduction and allows the selection of several informative features for modeling [47]. In radiomic analysis, it is necessary to combine the internal and external feature-selection methods to avoid redundancy and overfitting as much as possible.

\section{Modeling}

The ultimate goal of radiomics is to establish a practical and accurate model for predicting clinical outcomes (e.g., post-stroke death). In order to mine the full potential of existing datasets and explore the optimal model, clinical data should be added to the modeling process along with radiomic data. In addition, several machine-learning based modeling techniques can be employed. 
Table 1. Scopes and Specifications of Radiomic Studies in Stroke.

\begin{tabular}{|c|c|c|c|c|c|c|}
\hline Reference & Study Design & Image Modality & $\begin{array}{l}\text { No. of } \\
\text { Patients } \\
\end{array}$ & No. of Features (Type) & $\begin{array}{l}\text { Statistical } \\
\text { Method } \\
\end{array}$ & Clinical Utility \\
\hline \multicolumn{7}{|c|}{ Ischemic Stroke } \\
\hline $\begin{array}{l}\text { Oliveira et } \\
\text { al [56] }\end{array}$ & $\begin{array}{l}\text { Retrospective } \\
\text { Single-center }\end{array}$ & Non-contrast CT & 10 & 4 (texture) & $\begin{array}{l}\text { Univariate } \\
\text { analysis }\end{array}$ & $\begin{array}{l}\text { Diagnosis of acute } \\
\text { stroke lesions }\end{array}$ \\
\hline $\begin{array}{l}\text { Peter et al } \\
\text { [57] }\end{array}$ & $\begin{array}{l}\text { Retrospective } \\
\text { Single-center }\end{array}$ & Non-contrast CT & 139 & 18 (texture) & $\begin{array}{l}\text { Machine } \\
\text { learning }\end{array}$ & $\begin{array}{l}\text { Diagnosis of acute } \\
\text { stroke lesions }\end{array}$ \\
\hline $\begin{array}{l}\text { Sikio et al } \\
{[58]}\end{array}$ & $\begin{array}{l}\text { Retrospective } \\
\text { Single-center }\end{array}$ & T2W MRI & 30 & 4 (texture) & $\begin{array}{l}\text { Univariate } \\
\text { analysis }\end{array}$ & $\begin{array}{l}\text { Diagnosis of chronic } \\
\text { stroke lesions }\end{array}$ \\
\hline $\begin{array}{l}\text { Ortiz- } \\
\text { Ramon et al } \\
\text { [59] }\end{array}$ & $\begin{array}{l}\text { Prospective } \\
\text { Single-center }\end{array}$ & $\begin{array}{l}\text { MRI } \\
\text { (FLAIR, T1W and } \\
\text { T2W) }\end{array}$ & 100 & $\begin{array}{l}114 \text { (texture and } \\
\text { wavelet) }\end{array}$ & $\begin{array}{l}\text { Machine } \\
\text { learning }\end{array}$ & $\begin{array}{l}\text { Diagnosis of chronic } \\
\text { stroke lesions }\end{array}$ \\
\hline $\begin{array}{l}\text { Kassner et } \\
\text { al [60] }\end{array}$ & $\begin{array}{l}\text { Retrospective } \\
\text { Single-center }\end{array}$ & $\begin{array}{l}\text { Postcontrast T1W } \\
\text { MRI }\end{array}$ & 34 & 6 (intensity and texture) & $\begin{array}{l}\text { Logistic } \\
\text { regression }\end{array}$ & $\begin{array}{l}\text { Prediction of } \\
\text { hemorrhagic } \\
\text { transformation }\end{array}$ \\
\hline $\begin{array}{l}\text { Qiu et al } \\
\text { [61] }\end{array}$ & $\begin{array}{l}\text { Retrospective } \\
\text { Single-center }\end{array}$ & $\begin{array}{l}\text { Non-contrast } \\
\text { CT/CTA }\end{array}$ & 67 & $\begin{array}{l}326 \text { (shape, size, } \\
\text { intensity and texture) }\end{array}$ & $\begin{array}{l}\text { Machine } \\
\text { learning }\end{array}$ & $\begin{array}{l}\text { Prediction of early } \\
\text { recanalization }\end{array}$ \\
\hline $\begin{array}{l}\text { Tang et al } \\
{[62]}\end{array}$ & $\begin{array}{l}\text { Retrospective } \\
\text { Multi-center }\end{array}$ & $\begin{array}{l}\text { MRI } \\
\text { (CBF and ADC) }\end{array}$ & $\begin{array}{l}155 \\
(84+71)\end{array}$ & $\begin{array}{l}456 \text { (shape, intensity } \\
\text { and texture) }\end{array}$ & $\begin{array}{l}\text { LASSO } \\
\text { algorithm }\end{array}$ & $\begin{array}{l}\text { Evaluation of functional } \\
\text { outcomes }(7 d / 3 M)\end{array}$ \\
\hline $\begin{array}{l}\text { Cui et al } \\
{[63]}\end{array}$ & $\begin{array}{l}\text { Retrospective } \\
\text { Single-center }\end{array}$ & $\begin{array}{l}\text { MRI } \\
\text { (DWI and PWI) }\end{array}$ & $70(40+30)$ & $\begin{array}{l}251 \text { (intensity, texture } \\
\text { and high-order) }\end{array}$ & $\begin{array}{l}\text { Machine } \\
\text { learning }\end{array}$ & $\begin{array}{l}\text { Evaluation of functional } \\
\text { outcomes }(3 \mathrm{M})\end{array}$ \\
\hline $\begin{array}{l}\text { Betrouni et } \\
\text { al [64] }\end{array}$ & $\begin{array}{l}\text { Prospective } \\
\text { Multi-center }\end{array}$ & T1W MRI & 160 & $\begin{array}{l}11 \text { (intensity and } \\
\text { texture) }\end{array}$ & $\begin{array}{l}\text { Machine } \\
\text { learning }\end{array}$ & $\begin{array}{l}\text { Evaluation of cognitive } \\
\text { impairment }(6 \mathrm{M})\end{array}$ \\
\hline \multicolumn{7}{|l|}{$\mathrm{ICH}$} \\
\hline $\begin{array}{l}\text { Zhang et al } \\
{[66]}\end{array}$ & $\begin{array}{l}\text { Retrospective } \\
\text { Single-center }\end{array}$ & Non-contrast CT & $\begin{array}{l}261 \\
(180+81)\end{array}$ & $\begin{array}{l}576 \text { (shape, intensity, } \\
\text { texture, wavelet and } \\
\text { high-order) }\end{array}$ & $\begin{array}{l}\text { Machine } \\
\text { learning }\end{array}$ & $\begin{array}{l}\text { Diagnosis of AVM- } \\
\text { related hematomas }\end{array}$ \\
\hline $\begin{array}{l}\text { Barras et al } \\
{[70]}\end{array}$ & $\begin{array}{l}\text { Prospective } \\
\text { Multi-center }\end{array}$ & Non-contrast CT & 81 & 5 (intensity) & $\begin{array}{l}\text { Logistic } \\
\text { regression }\end{array}$ & $\begin{array}{l}\text { Prediction of early } \\
\text { hematoma expansion }\end{array}$ \\
\hline $\begin{array}{l}\text { Shen et al } \\
{[46]}\end{array}$ & $\begin{array}{l}\text { Retrospective } \\
\text { Single-center }\end{array}$ & Non-contrast CT & $\begin{array}{l}108 \\
(81+27)\end{array}$ & $\begin{array}{l}12 \text { (texture and high- } \\
\text { order) }\end{array}$ & $\begin{array}{l}\text { Logistic } \\
\text { regression }\end{array}$ & $\begin{array}{l}\text { Prediction of early } \\
\text { hematoma expansion }\end{array}$ \\
\hline $\begin{array}{l}\text { Ma et al } \\
\text { [47] }\end{array}$ & $\begin{array}{l}\text { Retrospective/ } \\
\text { Multi-center }\end{array}$ & Non-contrast CT & $\begin{array}{l}254 \\
(149+105)\end{array}$ & $\begin{array}{l}576 \text { (shape, intensity, } \\
\text { texture, wavelet and } \\
\text { high-order) }\end{array}$ & $\begin{array}{l}\text { LASSO } \\
\text { algorithm }\end{array}$ & $\begin{array}{l}\text { Prediction of early } \\
\text { hematoma expansion }\end{array}$ \\
\hline Li et al [48] & $\begin{array}{l}\text { Retrospective } \\
\text { Single-center }\end{array}$ & Non-contrast CT & 167 & $\begin{array}{l}1227 \text { (shape, size, } \\
\text { intensity, texture, } \\
\text { wavelet and high-order) }\end{array}$ & $\begin{array}{l}\text { Machine } \\
\text { learning }\end{array}$ & $\begin{array}{l}\text { Prediction of early } \\
\text { hematoma expansion }\end{array}$ \\
\hline $\begin{array}{l}\text { Xie et al } \\
{[49]}\end{array}$ & $\begin{array}{l}\text { Retrospective/ } \\
\text { Single-center }\end{array}$ & Non-contrast CT & $\begin{array}{l}251 \\
(177+74)\end{array}$ & $\begin{array}{l}1942 \text { (shape, size, } \\
\text { intensity, texture and } \\
\text { high-order) }\end{array}$ & $\begin{array}{l}\text { LASSO } \\
\text { algorithm }\end{array}$ & $\begin{array}{l}\text { Prediction of early } \\
\text { hematoma expansion }\end{array}$ \\
\hline $\begin{array}{l}\text { Yao et al } \\
{[31]} \\
\boldsymbol{S A H}\end{array}$ & $\begin{array}{l}\text { Retrospective } \\
\text { Single-center }\end{array}$ & Non-contrast CT & $\begin{array}{l}120 \\
(80+40)\end{array}$ & 300 (Not Specified) & $\begin{array}{l}\text { Machine } \\
\text { learning }\end{array}$ & $\begin{array}{l}\text { Prediction of early } \\
\text { edema area }\end{array}$ \\
\hline $\begin{array}{l}\text { Kanazawa } \\
\text { et al [71] }\end{array}$ & $\begin{array}{l}\text { Retrospective } \\
\text { Single-center } \\
\end{array}$ & Non-contrast CT & 40 & 4 (intensity) & $\begin{array}{l}\text { Logistic } \\
\text { regression } \\
\end{array}$ & $\begin{array}{l}\text { Prediction of functional } \\
\text { outcomes at discharge }\end{array}$ \\
\hline
\end{tabular}

Machine learning algorithms, such as support vector machines, artificial neural networks, and random forests, are supervised learning approaches that need to predefine a clinical label within a large number of training samples to train the model. Subsequently, the trained model is introduced to a testing set for performance evaluation. In contrast to supervised learning, unsupervised learning approaches (e.g., k-means clustering) can construct a model without clinical labels and using a limited sample size. Nevertheless, the performance of this model is limited by insufficiently labeled data. As a trade-off between high performance and low sample requirements, a growing number of semi-supervised learning models have recently been developed [18]. This method divides the modeling process into a supervised model training phase and an unsupervised feature learning phase, thus creating a balance between supervised and unsupervised learning. The choice of modeling techniques in radiomic 
analysis is important, in that different techniques may introduce discrepancies in model performance [51]. Therefore, multiple machine learning algorithms should be used for data mining, followed by the use of the optimal algorithm for modeling.

Validation is an indispensable step when constructing a prediction model for clinical applications. This could be carried out in either an internal or an external dataset. An externally validated model is considered more credible than an internally validated model, since independently obtained data can strengthen the validation [52]. Model performance is typically characterized by 2 attributes: discrimination and calibration [53]. Discrimination refers to how well the model distinguishes between those who are at high risk of a clinical event and those who are at low risk, and is often measured by means of receiver operating characteristic (ROC) curves, of which the area under the curve (AUC), sensitivity, and specificity are the 3 most commonly used indices. Calibration is a measure of agreement between the model's predicted risk and observed risk, which can be reported in the manner of visuals (calibration curve) or statistical tests (e.g., the Hosmer-Lemeshow test). A reliable and applicable model should have both favorable discrimination and calibration and should exhibit statistical consistency between the training and validation datasets.

\section{Applications of radiomics in stroke}

With the advent of radiomics, additional information provided by quantitative image analysis has become an important supplement to traditional radiologic characteristics, accelerating the development of precision diagnosis and treatment in oncology [18]. Over the past few years, researchers have attempted to apply radiomics to stroke in the hope of gaining benefits similar to those obtained in cancer management. Radiomic data are expected to improve early decision-making for poststroke patients. Currently, some progress has been made in both fields of ischemic and hemorrhagic strokes, and these applications seem to fall into 3 aspects: diagnosis of stroke lesion, prediction of early outcome, and long-term prognosis evaluation (Table 1).

\subsection{Applications in ischemic stroke}

To distinguish ischemic stroke from transient ischemic attack, an updated definition of ischemic stroke is an acute episode of neurologic deficit lasting longer than $24 \mathrm{~h}$, or the presence of any imaging evidence (CT and/or MRI) of infarction directly related to the symptoms [1]. Ischemic stroke is the primary subtype of stroke that accounts for approximately $85 \%$ of all cases, and one-third of stroke patients will be permanently disabled [54].

\section{Diagnosis of stroke lesions}

Clinically, NCCT remains the first choice for patients with suspected stroke because it is efficient, non-invasive, and low in cost. Thrombolytic treatment with tissue plasminogen activator (tPA) in ischemic stroke patients benefits markedly from intravenous administration within $4.5 \mathrm{~h}$ after symptom onset [55], which relies on early identification of stroke lesions by NCCT scans. In the acute phase, however, the changes in the ischemic area on NCCT images are often too subtle to be captured visually. In a study of 10 sex-and age-matched subjects (5 patients and 5 controls), Oliveira et al. [56] performed a quantitative texture analysis to distinguish healthy tissue from regions affected by AIS. They found that the graylevel co-occurrence matrix (GLCM)-based tissue texture parameters were significantly different between patients and controls, and the most discriminative feature was angular second moment. In another study of 139 patients with hyperacute ischemic stroke $(<8 \mathrm{~h})$, the authors identified 6 texture features from NCCT images that could differentiate ischemic lesions from their contralateral normal tissue [57]. The classification model, constructed by 3 supervised machine learning algorithms (i.e., support vector machine, decision trees, and adaboost) achieved an average AUC of 0.82. In addition, they found that the size of the stroke lesion and classifier type did not affect the model performance.

Two other studies have investigated the diagnostic value of radiomics in stroke lesions using MR images. Sikio et al [58] evaluated 30 patients with chronic right hemisphere infarction and found that GLCM texture features derived from T2-weighted MR images were capable of revealing changes in both ischemic lesions and the ipsilateral structure outside the lesion (i.e., centrum semiovale). The ischemic region had lower homogeneity texture parameters than the unaffected side, but with relatively high values of complexity and randomness. Additionally, they found a close association between texture and diffusion tensor imaging (DTI) parameters (Pearson's $r>0.5$ ) in the ipsilateral mesencephalon and thereby concluded that texture analysis might be a useful tool for assisting the DTI method in the detection of chronic ischemic lesions. Ortiz-Ramon and colleagues [59] used multimodal MRI data of different brain tissues (i.e., white matter and subcortical structures) from 100 elderly individuals to explore whether radiomic analysis could distinguish patients who had a prior ischemic stroke from a healthy population that was stroke-free. They found that radiomic features, including texture and wavelet, could robustly identify the presence of previous stroke lesions with favorable discrimination (AUC > 0.7), irrespective of MRI sequence used, tissue type, and stroke subtype. 


\section{Prediction of early outcomes}

The extra benefit of intravenous tPA for AIS comes from exclusion of patients with a high risk of secondary intracranial hemorrhage, which is a serious complication, primarily due to damage of the blood-brain barrier. Kassner et al [60] conducted a comparative study to assess the predictive ability for early hemorrhagic transformation after AIS $(<72 \mathrm{~h})$ between texture parameters and visual enhancement score in postcontrast T1-weighted MR images. Texture features (contrast and correlation) were found to be more predictive of hemorrhagic transformation than visual evidence of enhancement (AUC < 0.6), with an AUC > 0.75. This result is promising, because texture analysis may help select eligible patients who are most likely to benefit from thrombolytic treatment. For thrombus in the internal carotid artery and M1 middle cerebral artery segment, recanalization with thrombolytics within these proximal intracranial arterial segments is rare. Qiu et al [61] performed a radiomic analysis to predict early recanalization after proximal occlusion in large vessels in 67 AIS patients administered intravenous alteplase. They concluded that the combination of radiomic features from NCCT, CTA, and radiomic changes (i.e., CTA-NCCT) was more predictive of early recanalization $(\mathrm{AUC}=0.85)$, compared with solely conventional thrombus imaging characteristics, such as length, volume, or permeability.

\section{Evaluation of long-term prognosis}

Accurate identification of a salvageable penumbra promises to improve decision-making during post-stroke management and extend the therapeutic time window. A multicenter study $(n=155)$ conducted by Tang et al [62] applied radiomic analysis to quantify the penumbra and core area from both the apparent diffusion coefficient and cerebral blood flow maps in patients with AIS $(<9 \mathrm{~h})$. In the external dataset, the constructed radiomics nomogram could strongly predict favorable clinical outcomes at 7 days and at 3 months, with AUCs of 0.88 and 0.77, respectively. Thus, researchers concluded that radiomics has the potential to select patients for thrombolysis beyond the current time window. Another study based on multimodal MR images of AIS patients confirmed the prognostic value of radiomic features in predicting 3month outcomes using a feature selection strategy combining redundancy reduction and informative degree evaluation [63].

Betrouni et al [64] found that texture features of MR images (i.e., kurtosis and inverse difference moment) in the hippocampus and entorhinal cortex at $72 \mathrm{~h}$ after stroke onset were significantly different between patients with and without 6-month cognitive impairment (CI). The prediction model built using a support vector machine showed excellent discrimination ability for CI (AUC > 0.9 ). This result was further confirmed in a rat model of middle cerebral artery transient occlusion, in which there was a significant correlation between texture features and neural density in the hippocampus contralateral to the ischemic region. These associations are based on the hypothesis that the mild neuron loss involved in poststroke CI could be captured at an early stage by measuring changes in image gray values. The results of this study are relatively reliable because of synthesized clinical and preclinical evidence and indicate that the MR texture features may be an early imaging marker for screening of long-term CI in patients with AIS.

\subsection{Applications in hemorrhagic stroke}

Hemorrhagic stroke subtypes include ICH and SAH, both of which are not caused by trauma. Although hemorrhagic stroke accounts for only a small proportion of stroke cases (ca. 15\%), its associated disability and mortality are high. It is reported that ICH alone has a fatality rate of $40 \%$ at 1 month [65].

\section{Diagnosis of stroke lesions}

In clinical scenarios, the diagnosis of ICH caused by arteriovenous malformation (AVM) relies to a large extent on the CTA technique, since AVM-related hematomas are difficult to distinguish on NCCT images from those triggered by hypertension or cerebral amyloid angiopathy. However, angiography entails a large investment of time and resources, and may not be widely available or routinely performed in primary medical institutions. Early and accurate diagnosis of AVM-related hematomas is crucial for guiding treatment decisions, for instance, deciding whether or not to embolize the nidus to avoid rebleeding. Zhang and colleagues [66] performed a radiomics study to differentiate between acute $\mathrm{ICH}(<6$ h) of diverse etiologies on NCCT. The hypothesis driving this research is that AVM-related hematomas embedded in malformed vasculature are more heterogeneous in composition and could be identified through quantitative radiomic analysis. After integrating optimal feature selection and modeling algorithms, the established NCCT radiomics model could accurately diagnose AVM-ICH in the validation cohort $(\mathrm{n}=81)$, with an AUC of 0.95 , sensitivity of $88 \%$, and specificity of $93 \%$. In addition, radiomic analysis showed superior diagnostic performance to subjective assessment by interventional radiologists with different levels of work experience.

Prediction of early outcomes 
Hematoma expansion (HE) following ICH reflects an active bleeding process, which is strongly associated with early neurological deterioration and poor long-term prognosis and is an appealing treatment target in clinical trials $[67,68]$. In addition to the currently available clinical and radiologic risk factors [69], a growing body of evidence in radiomics has shown promise for predicting HE [46-49, 70].

In 2013, Barras et al [70] conducted the first radiomics study on NCCT images of acute ICH patients $(<3 \mathrm{~h})$ and identified a histogram intensity feature (i.e., coefficient of variation) most relevant to HE. In 2018, Shen et al [46] used the Laplacian of Gaussian bandpass filter to extract a series of coarse to fine texture features from images to predict early HE, with AUC reaching 0.92 . Recently, the favorable performance of the radiomics model for $\mathrm{HE}$ was confirmed in a multicenter study $(\mathrm{n}=$ 254) [47], wherein ICH data from 4 independent medical centers were analyzed. In another comparative study evaluating 251 patients with acute $\mathrm{ICH}$, the radiomics model was found to be superior to the radiological model that incorporates conventional NCCT markers (AUC, 0.9 versus 0.8) [49]. Notably, the interpretation of results among these studies is limited by different HE definitions (>6 mL, > $12.5 \mathrm{~mL}$, or > 33\%) and the non-standardized follow-up NCCT timing for HE detection. Besides the predictive value of ICH growth, radiomic signature has also been reported to be highly predictive of the edema area around the basal ganglia hematoma at an early stage [31].

\section{Evaluation of long-term prognosis}

Clinical data regarding the evaluation of prognosis in hemorrhagic stroke using radiomic analysis are scarce. However, recently, a pilot study conducted by Kanazawa et al [71] explored the feasibility of quantitative texture analysis of NCCT images in predicting clinical outcomes in 40 patients with aneurysmal SAH. They found that the mean CT value of subarachnoid bleeding at the level of the basal cisterna was the only texture feature independently associated with delayed cerebral ischemia and prognosis at discharge. At the optimal cutoff value of $53 \mathrm{HU}$, this parameter could predict poor functional outcomes $(\mathrm{mRS} \geq 3)$ with a high specificity of $91.7 \%$. Due to their qualitative nature, radiological markers such as the Fisher, Hijdra, and SEBES grading systems for measuring SAH severity, are susceptible to interobserver variability $[72,73]$. This work is an initial step toward the development of a quantitative radiomics tool for the assessment of SAH prognosis on NCCT images. For ischemic and hemorrhagic stroke applications, the difference between CT- and MRI-radiomics is presented in Table 2.

Table 2. Comparison of CT- and MRI-radiomics in Stroke Application.

\begin{tabular}{|c|c|c|c|c|}
\hline Neuroimaging & Technique & Study Population & Research Objective & Pros and Cons \\
\hline $\mathrm{CT}$ & $\begin{array}{l}\text { Non-contrast CT } \\
\text { CTA }\end{array}$ & $\begin{array}{l}\text { Acute ischemic } \\
\text { stroke } \\
\text { Intracerebral } \\
\text { hemorrhage } \\
\text { Subarachnoid } \\
\text { hemorrhage }\end{array}$ & $\begin{array}{l}\text { Diagnosis of acute } \\
\text { stroke lesions } \\
\text { Prediction of early } \\
\text { outcomes }\end{array}$ & $\begin{array}{l}\text { Convenient for image data acquisition and } \\
\text { transformation } \\
\text { Difficulty in ROI segmentation of acute } \\
\text { ischemic stroke } \\
\text { Limited source of radiomics information } \\
\text { from lesions } \\
\text { More interpretable for radiomic features }\end{array}$ \\
\hline MRI & $\begin{array}{l}\text { T1W/T2W/FLAIR } \\
\text { Postcontrast T1W } \\
\text { DWI (ADC) } \\
\text { PWI (CBF) }\end{array}$ & $\begin{array}{l}\text { Acute ischemic } \\
\text { stroke } \\
\text { Chronic ischemic } \\
\text { stroke }\end{array}$ & $\begin{array}{l}\text { Diagnosis of chronic } \\
\text { stroke lesions } \\
\text { Evaluation of long- } \\
\text { term prognosis }\end{array}$ & $\begin{array}{l}\text { Inconvenience in archiving and } \\
\text { communication caused by relatively large } \\
\text { image data } \\
\text { Accurate 3D-ROI delineation of ischemic } \\
\text { lesions } \\
\text { Entail resampling intensity values for } \\
\text { multimodal images } \\
\text { Lack of interpretability of radiomic features }\end{array}$ \\
\hline
\end{tabular}

CTA, computed tomography angiography; DWI, diffusion-weighted imaging; ADC, apparent diffusion coefficient; PWI, perfusion-weighted imaging; $\mathrm{CBF}$, cerebral blood flow; ROI, region of interest.

\section{Challenges and Future Directions}

Several challenges currently exist around radiomics, facing its widespread use in stroke management. One of the most critical challenges is the lack of reproducibility among extracted radiomic features, which arises primarily from the variability in radiomic workflow. Since radiomics studies can be performed either on open-source software platforms (e.g., MaZda or IBEX) or with inhouse developed tools in the MATLAB environment, the pre- and post-processing techniques, the segmentation method for the ROI, and the type and quantity of extracted 
radiomic features are significantly different among these investigations. This contributes to difficulties in reproduction and comparisons between studies, as well as in the integration of results for meta-analysis. To normalize the reporting process and improve repeatability, investigators have proposed the radiomics quality score (RQS) to help ascertain whether a radiomics study conforms to best-practice procedures [20]. More recently, the image biomarker standardization initiative (IBSI) has also been put forward, offering a standardized general image processing workflow for quantitative radiomic analysis [74]. Future radiomics studies need to be carried out in the light of these reporting guidelines.

As mentioned earlier, imaging protocols highly affect the stability of radiomic features and, thus, may be another factor that introduces non-reproducibility in radiomics. It has recently been reported that $\mathrm{CT}$ image acquisition and reconstruction parameters could cause variation in the values of a considerable number of features $[22,25,26]$. This is understandable, considering that a greater slice thickness may increase the error in ROI delineation due to the partial volume effect. Although some efforts have been made to improve the robustness of radiomic features, such as the use of preprocessing techniques of filtration or gray-level normalization, achievements are extremely limited, particularly in stroke. The Quantitative Imaging Network (QIN) is a cooperative project initiated by the National Institutes of Health (NIH) in an effort to develop new quantitative imaging tools and methods for cancer management in clinical trials; one of its goals is to promote the standardization of quantitative imaging protocols in cancer [75]. For radiomics studies of stroke, there is a pressing need to develop standardized MRI and CT scanning protocols that are widely acceptable in the research community.

While NCCT-based radiomic features have the ability to identify acute infarcted tissue, the information they capture is confined to a part of the lesion in a single slice (2D-radiomics) [56, 57]. It is challenging to perform a volumetric assessment for stroke lesions that cannot be visually perceived. Although MR diffusion and perfusion imaging perform well in this regard, NCCT is increasingly favored in routine practice because of its speed, low cost, and lack of contraindications. Therefore, future research should focus on developing semi- or full-automatic segmentation tools for acute stroke lesions on NCCT images. Interestingly, 3D-volumetric measurements in ICH and SAH have achieved automatic segmentation with the aid of machine learning $[39,76]$. The next step is to determine the best way to integrate radiomic data from multiple hemorrhage lesions. For example, it remains unclear whether radiomic features derived from blood clots in different cerebral cisterns or at multiple locations (e.g., brain stem and cerebellum) should be summed, averaged, or weighted. This issue exists widely in the radiomic analysis of other lesion types, and thus, the solution is of great significance.

Another major challenge in radiomic analysis is the need for large-scale data. Statistically adequate radiomic data are conducive to generating a classifier model with high robustness and generalization. However, the creation of databases is time-consuming because of the collection of medical images and 3D-ROIs segmentation. Although deep learning algorithms based on convolutional neural networks can automatically extract features from an unsegmented image, the requirements for expensive hardware and immense volumes of annotated data have limited their applicability [77-79]. Consequently, data sharing among different research organizations and medical centers is crucial in radiomics $[13,16]$. Currently, most of the available radiomic evidence regarding stroke is derived from retrospective and single-center studies, which leads to an unstable association of radiomic features with clinical events, due to selection bias. For instance, the type of radiomic features that could predict early HE of acute ICH differed markedly across studies [47-49]. Shared databases consisting of radiomic data and other pertinent medical information (e.g., clinical or demographic data) from multiple centers, after removing the data management hurdle, may serve as an external tool for validating the credibility of existing results and eventually promote the standardization of radiomics research in stroke around the world.

\section{Conclusions}

With advances in computational technologies, radiomic analyses of stroke neuroimages have been applied successfully to NCCT and MRI scans. The derived radiomic signature could potentially be used to diagnose stroke lesions, predict early transformation, and evaluate long-term prognosis after stroke onset. Despite these promising results, various challenges need to be addressed before its widespread use as a clinical tool. These challenges seem to arise from the reproducibility of study results, standardization of protocols in radiomic workflow, and data sharing among different medical institutions. By mining the full potential of radiomics in the field of stroke, it is expected to optimize secondary prevention strategies and facilitate the development of personalized precision medicine in post-stroke patients.

\section{Conflict of Interest}

The authors declare no conflict of interest. 


\section{Acknowledgments}

This work was supported by the Science and Technology Planning Projects of Wenzhou (Grant No. Y20180112), Health Foundation for Creative Talents in Zhejiang Province, China (No.: 2016) and Project Foundation for the College Young and Middle-aged Academic Leader of Zhejiang Province, China (No.: 2017).

\section{References}

[1] Sacco RL, Kasner SE, Broderick JP, Caplan LR, Connors JJ, Culebras A, et al (2013). An updated definition of stroke for the 21st century: a statement for healthcare professionals from the American Heart Association/American Stroke Association. Stroke, 44:2064-2089.

[2] Rajsic S, Gothe H, Borba HH, Sroczynski G, Vujicic J, Toell T, et al (2018). Economic burden of stroke: a systematic review on post-stroke care. The European Journal of Health Economics, 20:107-134.

[3] Johnson CO, Nguyen M, Roth GA, Nichols E, Alam T, Abate D, et al (2019). Global, regional, and national burden of stroke, 1990-2016: a systematic analysis for the Global Burden of Disease Study 2016. Lancet Neurology, 18:439-458.

[4] Buckler AJ, Bresolin L, Dunnick NR, Sullivan DC, Group (2011). A collaborative enterprise for multistakeholder participation in the advancement of quantitative imaging. Radiology, 258:906-914.

[5] Lambin P, van Stiphout RG, Starmans MH, RiosVelazquez E, Nalbantov G, Aerts HJ, et al (2013). Predicting outcomes in radiation oncology-multifactorial decision support systems. Nat Rev Clin Oncol, 10:27-40.

[6] Badhiwala JH, Nassiri F, Alhazzani W, Selim MH, Farrokhyar F, Spears J, et al (2015). Endovascular Thrombectomy for Acute Ischemic Stroke. JAMA, 314:1832-1843.

[7] Goyal M, Menon BK, van Zwam WH, Dippel DWJ, Mitchell PJ, Demchuk AM, et al (2016). Endovascular thrombectomy after large-vessel ischaemic stroke: a meta-analysis of individual patient data from five randomised trials. Lancet, 387:1723-1731.

[8] Davis S, Campbell B, Christensen S, Ma H, Desmond P, Parsons M, et al (2012). Perfusion/Diffusion mismatch is valid and should be used for selecting delayed interventions. Transl Stroke Res, 3:188-197.

[9] Morotti A, Boulouis G, Romero JM, Brouwers HB, Jessel MJ, Vashkevich A, et al (2017). Blood pressure reduction and noncontrast $\mathrm{CT}$ markers of intracerebral hemorrhage expansion. Neurology, 89:548-554.

[10] Morotti A, Brouwers HB, Romero JM, Jessel MJ, Vashkevich A, Schwab K, et al (2017). Intensive Blood Pressure Reduction and Spot Sign in Intracerebral Hemorrhage: A Secondary Analysis of a Randomized Clinical Trial. JAMA Neurol, 74:950-960.

[11] Meretoja A, Churilov L, Campbell BC, Aviv RI, Yassi N, Barras C, et al (2014). The spot sign and tranexamic acid on preventing ICH growth--AUStralasia Trial (STOPAUST): protocol of a phase II randomized, placebocontrolled, double-blind, multicenter trial. Int J Stroke, 9:519-524

[12] Delcourt C, Zhang S, Arima H, Sato S, Al-Shahi Salman $\mathrm{R}$, Wang $\mathrm{X}$, et al (2016). Significance of Hematoma Shape and Density in Intracerebral Hemorrhage: The Intensive Blood Pressure Reduction in Acute Intracerebral Hemorrhage Trial Study. Stroke, 47:12271232.

[13] Avanzo M, Stancanello J, El Naqa I (2017). Beyond imaging: The promise of radiomics. Phys Med, 38:122139.

[14] Yip SS, Aerts HJ (2016). Applications and limitations of radiomics. Phys Med Biol, 61:R150-166.

[15] Lambin P, Rios-Velazquez E, Leijenaar R, Carvalho S, van Stiphout RG, Granton P, et al (2012). Radiomics: extracting more information from medical images using advanced feature analysis. Eur J Cancer, 48:441-446.

[16] Gillies RJ, Kinahan PE, Hricak H (2016). Radiomics: Images Are More than Pictures, They Are Data. Radiology, 278:563-577.

[17] Aerts HJ, Velazquez ER, Leijenaar RT, Parmar C, Grossmann P, Carvalho S, et al (2014). Decoding tumour phenotype by noninvasive imaging using a quantitative radiomics approach. Nat Commun, 5:4006-4006.

[18] Liu Z, Wang S, Dong D, Wei J, Fang C, Zhou X, et al (2019). The Applications of Radiomics in Precision Diagnosis and Treatment of Oncology: Opportunities and Challenges. Theranostics, 9:1303-1322.

[19] Gatenby RA, Grove O, Gillies RJ (2013). Quantitative Imaging in Cancer Evolution and Ecology. Radiology, 269:8-14.

[20] Lambin P, Leijenaar RTH, Deist TM, Peerlings J, de Jong EEC, van Timmeren J, et al (2017). Radiomics: the bridge between medical imaging and personalized medicine. Nat Rev Clin Oncol, 14:749-762.

[21] Heit JJ, Iv M, Wintermark M (2017). Imaging of Intracranial Hemorrhage. J Stroke, 19:11-27.

[22] Larue RT, Defraene G, De Ruysscher D, Lambin P, van Elmpt W (2017). Quantitative radiomics studies for tissue characterization: a review of technology and methodological procedures. Br J Radiol, 90:20160665.

[23] Kumar V, Gu Y, Basu S, Berglund A, Eschrich SA, Schabath MB, et al (2012). Radiomics: the process and the challenges. Magn Reson Imaging, 30:1234-1248.

[24] Midya A, Chakraborty J, Gönen M, Do RKG, Simpson AL (2018). Influence of CT acquisition and reconstruction parameters on radiomic feature reproducibility. Journal of medical imaging (Bellingham, Wash.), 5:011020-011020.

[25] Meyer M, Ronald J, Vernuccio F, Nelson RC, RamirezGiraldo JC, Solomon J, et al (2019). Reproducibility of CT Radiomic Features within the Same Patient: Influence of Radiation Dose and CT Reconstruction Settings. Radiology, 293:583-591.

[26] Berenguer R, Pastor-Juan MDR, Canales-Vázquez J, Castro-García M, Villas MV, Mansilla Legorburo F, et al (2018). Radiomics of CT Features May Be Nonreproducible and Redundant: Influence of CT 
Acquisition Parameters. Radiology, 288:407-415.

[27] Mackin D, Fave X, Zhang L, Fried D, Yang J, Taylor B, et al (2015). Measuring Computed Tomography Scanner Variability of Radiomics Features. Invest Radiol, 50:757-765.

[28] Larue R, van Timmeren JE, de Jong EEC, Feliciani G, Leijenaar RTH, Schreurs WMJ, et al (2017). Influence of gray level discretization on radiomic feature stability for different CT scanners, tube currents and slice thicknesses: a comprehensive phantom study. Acta Oncol, 56:1544-1553.

[29] Shafiq-Ul-Hassan M, Latifi K, Zhang G, Ullah G, Gillies R, Moros E (2018). Voxel size and gray level normalization of $\mathrm{CT}$ radiomic features in lung cancer. Sci Rep, 8:10545.

[30] Shafiq-Ul-Hassan M, Zhang GG, Latifi K, Ullah G, Hunt DC, Balagurunathan $\mathrm{Y}$, et al (2017). Intrinsic dependencies of CT radiomic features on voxel size and number of gray levels. Med Phys, 44:1050-1062.

[31] Yao X, Liao L, Han Y, Wei T, Wu H, Wang Y, et al (2019). Computerized Tomography Radiomics Features Analysis for Evaluation of Perihematomal Edema in Basal Ganglia Hemorrhage. J Craniofac Surg, 30:e768e771.

[32] Lubner MG, Smith AD, Sandrasegaran K, Sahani DV, Pickhardt PJ (2017). CT Texture Analysis: Definitions, Applications, Biologic Correlates, and Challenges. Radiographics, 37:1483-1503.

[33] Ng F, Kozarski R, Ganeshan B, Goh V (2013). Assessment of tumor heterogeneity by $\mathrm{CT}$ texture analysis: can the largest cross-sectional area be used as an alternative to whole tumor analysis? Eur J Radiol, 82:342-348

[34] Mitra J, Bourgeat P, Fripp J, Ghose S, Rose S, Salvado $\mathrm{O}$, et al (2014). Lesion segmentation from multimodal MRI using random forest following ischemic stroke. NeuroImage, 98:324-335.

[35] McKinley R, Hani L, Gralla J, El-Koussy M, Bauer S, Arnold M, et al (2017). Fully automated stroke tissue estimation using random forest classifiers (FASTER). J Cereb Blood Flow Metab, 37:2728-2741.

[36] Subbanna NK, Rajashekar D, Cheng B, Thomalla G, Fiehler J, Arbel T, et al (2019). Stroke Lesion Segmentation in FLAIR MRI Datasets Using Customized Markov Random Fields. Front Neurol, 10:541-541.

[37] Kamnitsas K, Ledig C, Newcombe VFJ, Simpson JP, Kane AD, Menon DK, et al (2017). Efficient multi-scale 3D CNN with fully connected CRF for accurate brain lesion segmentation. Med Image Anal, 36:61-78.

[38] Xu X, Chen X, Zhang J, Zheng Y, Sun G, Yu X, et al (2014). Comparison of the Tada formula with software slicer: precise and low-cost method for volume assessment of intracerebral hematoma. Stroke, 45:34333435 .

[39] Scherer M, Cordes J, Younsi A, Sahin Y-A, Götz M, Möhlenbruch M, et al (2016). Development and Validation of an Automatic Segmentation Algorithm for Quantification of Intracerebral Hemorrhage. Stroke, 47:2776-2782.
[40] Hassani C, Varghese BA, Nieva J, Duddalwar V (2019). Radiomics in Pulmonary Lesion Imaging. AJR Am J Roentgenol, 212:497-504.

[41] Haralick RM, Shanmugam K, Dinstein I (1973). Textural Features for Image Classification. Ieee Transactions on Systems Man and Cybernetics, Smc3:610-621.

[42] Castellano G, Bonilha L, Li LM, Cendes F (2004). Texture analysis of medical images. Clin Radiol, 59:1061-1069.

[43] Scalco E, Rizzo G (2017). Texture analysis of medical images for radiotherapy applications. $\mathrm{Br} \mathrm{J}$ Radiol, 90:20160642.

[44] Porwik P, Lisowska A (2004). The Haar-wavelet transform in digital image processing: its status and achievements. Machine Graphics \& Vision, 13:79-98.

[45] Davnall F, Yip CSP, Ljungqvist G, Selmi M, Ng F, Sanghera B, et al (2012). Assessment of tumor heterogeneity: an emerging imaging tool for clinical practice? Insights into Imaging, 3:573-589.

[46] Shen Q, Shan Y, Hu Z, Chen W, Yang B, Han J, et al (2018). Quantitative parameters of CT texture analysis as potential markersfor early prediction of spontaneous intracranial hemorrhage enlargement. Eur Radiol, 28:4389-4396

[47] Ma C, Zhang Y, Niyazi T, Wei J, Guocai G, Liu J, et al (2019). Radiomics for predicting hematoma expansion in patients with hypertensive intraparenchymal hematomas. Eur J Radiol, 115:10-15.

[48] Li H, Xie Y, Wang X, Chen F, Sun J, Jiang X (2019). Radiomics features on non-contrast computed tomography predict early enlargement of spontaneous intracerebral hemorrhage. Clin Neurol Neurosurg, 185:105491.

[49] Xie H, Ma S, Wang X, Zhang X (2020). Noncontrast computer tomography-based radiomics model for predicting intracerebral hemorrhage expansion: preliminary findings and comparison with conventional radiological model. Eur Radiol, 30:87-98.

[50] Bagherzadeh-Khiabani F, Ramezankhani A, Azizi F, Hadaegh F, Steyerberg EW, Khalili D (2016). A tutorial on variable selection for clinical prediction models: feature selection methods in data mining could improve the results. J Clin Epidemiol, 71:76-85.

[51] Parmar C, Grossmann P, Bussink J, Lambin P, Aerts H (2015). Machine Learning methods for Quantitative Radiomic Biomarkers. Sci Rep, 5:13087.

[52] Debray TP, Vergouwe Y, Koffijberg H, Nieboer D, Steyerberg EW, Moons KG (2015). A new framework to enhance the interpretation of external validation studies of clinical prediction models. J Clin Epidemiol, 68:279289.

[53] Alba AC, Agoritsas T, Walsh M, Hanna S, Iorio A, Devereaux PJ, et al (2017). Discrimination and Calibration of Clinical Prediction Models: Users' Guides to the Medical Literature. JAMA, 318:1377-1384.

[54] Musuka TD, Wilton SB, Traboulsi M, Hill MD (2015). Diagnosis and management of acute ischemic stroke: speed is critical. CMAJ, 187:887-893.

[55] Wardlaw JM, Murray V, Berge E, del Zoppo G, Sandercock P, Lindley RL, et al (2012). Recombinant 
tissue plasminogen activator for acute ischaemic stroke: an updated systematic review and meta-analysis. Lancet, 379:2364-2372.

[56] Oliveira MS, Fernandes PT, Avelar WM, Santos SL, Castellano G, Li LM (2009). Texture analysis of computed tomography images of acute ischemic stroke patients. Braz J Med Biol Res, 42:1076-1079.

[57] Peter R, Korfiatis P, Blezek D, Oscar Beitia A, StepanBuksakowska I, Horinek D, et al (2017). A quantitative symmetry-based analysis of hyperacute ischemic stroke lesions in noncontrast computed tomography. Med Phys, 44:192-199.

[58] Sikio M, Kolhi P, Ryymin P, Eskola HJ, Dastidar P (2015). MRI Texture Analysis and Diffusion Tensor Imaging in Chronic Right Hemisphere Ischemic Stroke. J Neuroimaging, 25:614-619.

[59] Ortiz-Ramon R, Valdes Hernandez MDC, GonzalezCastro V, Makin S, Armitage PA, Aribisala BS, et al (2019). Identification of the presence of ischaemic stroke lesions by means of texture analysis on brain magnetic resonance images. Comput Med Imaging Graph, 74:1224.

[60] Kassner A, Liu F, Thornhill RE, Tomlinson G, Mikulis DJ (2009). Prediction of hemorrhagic transformation in acute ischemic stroke using texture analysis of postcontrast T1-weighted MR images. J Magn Reson Imaging, 30:933-941.

[61] Qiu W, Kuang H, Nair J, Assis Z, Najm M, McDougall C, et al (2019). Radiomics-Based Intracranial Thrombus Features on CT and CTA Predict Recanalization with Intravenous Alteplase in Patients with Acute Ischemic Stroke. AJNR Am J Neuroradiol, 40:39-44.

[62] Tang TY, Jiao Y, Cui Y, Zhao DL, Zhang Y, Wang Z, et al (2020). Penumbra-based radiomics signature as prognostic biomarkers for thrombolysis of acute ischemic stroke patients: a multicenter cohort study. J Neurol:10.1007/s00415-00020-09713-00417.

[63] Cui H, Wang X, Bian Y, Song S, Feng DD (2018). Ischemic stroke clinical outcome prediction based on image signature selection from multimodality data. Conference proceedings : ... Annual International Conference of the IEEE Engineering in Medicine and Biology Society. IEEE Engineering in Medicine and Biology Society. Annual Conference, 2018:722-725.

[64] Betrouni N, Yasmina M, Bombois S, Petrault M, Dondaine T, Lachaud C, et al (2019). Texture Features of Magnetic Resonance Images: an Early Marker of Poststroke Cognitive Impairment. Transl Stroke Res:10.1007/s12975-12019-00746-12973.

[65] Keep RF, Hua Y, Xi G (2012). Intracerebral haemorrhage: mechanisms of injury and therapeutic targets. Lancet Neurol, 11:720-731.

[66] Zhang Y, Zhang B, Liang F, Liang S, Zhang Y, Yan P, et al (2019). Radiomics features on non-contrast-enhanced CT scan can precisely classify AVM-related hematomas from other spontaneous intraparenchymal hematoma types. Eur Radiol, 29:2157-2165.

[67] Brouwers HB, Greenberg SM (2013). Hematoma expansion following acute intracerebral hemorrhage. Cerebrovasc Dis, 35:195-201.

[68] Dowlatshahi D, Demchuk AM, Flaherty ML, Ali M, Lyden PL, Smith EE, et al (2011). Defining hematoma expansion in intracerebral hemorrhage: relationship with patient outcomes. Neurology, 76:1238-1244.

[69] Al-Shahi Salman R, Frantzias J, Lee RJ, Lyden PD, Battey TWK, Ayres AM, et al (2018). Absolute risk and predictors of the growth of acute spontaneous intracerebral haemorrhage: a systematic review and meta-analysis of individual patient data. Lancet Neurol, 17:885-894.

[70] Barras CD, Tress BM, Christensen S, Collins M, Desmond PM, Skolnick BE, et al (2013). Quantitative CT densitometry for predicting intracerebral hemorrhage growth. AJNR Am J Neuroradiol, 34:1139-1144.

[71] Kanazawa T, Takahashi S, Minami Y, Jinzaki M, Toda M, Yoshida K (2020). Early prediction of clinical outcomes in patients with aneurysmal subarachnoid hemorrhage using computed tomography texture analysis. J Clin Neurosci, 71:144-149.

[72] Woo PYM, Tse TPK, Chan RSK, Leung LNY, Liu SKK, Leung AYT, et al (2017). Computed tomography interobserver agreement in the assessment of aneurysmal subarachnoid hemorrhage and predictors for clinical outcome. J Neurointerv Surg, 9:1118-1124.

[73] Ahn SH, Savarraj JP, Pervez M, Jones W, Park J, Jeon $\mathrm{SB}$, et al (2018). The Subarachnoid Hemorrhage Early Brain Edema Score Predicts Delayed Cerebral Ischemia and Clinical Outcomes. Neurosurgery, 83:137-145.

[74] Zwanenburg A, Leger S, Valli eres M, S Lo Image biomarker standardisation initiative. arXiv preprint arXiv:1612.07003.

[75] Clarke LP, Nordstrom RJ, Zhang H, Tandon P, Zhang Y, Redmond $\mathrm{G}$, et al (2014). The Quantitative Imaging Network: NCI's Historical Perspective and Planned Goals. Transl Oncol, 7:1-4.

[76] Boers AM, Zijlstra IA, Gathier CS, van den Berg R, Slump CH, Marquering HA, et al (2014). Automatic quantification of subarachnoid hemorrhage on noncontrast CT. AJNR Am J Neuroradiol, 35:2279-2286.

[77] Litjens G, Kooi T, Bejnordi BE, Setio AAA, Ciompi F, Ghafoorian M, et al (2017). A survey on deep learning in medical image analysis. Med Image Anal, 42:60-88.

[78] Feng R, Badgeley M, Mocco J, Oermann EK (2018). Deep learning guided stroke management: a review of clinical applications. J Neurointerv Surg, 10:358-362.

[79] Zaharchuk G, Gong E, Wintermark M, Rubin D, Langlotz CP (2018). Deep Learning in Neuroradiology. AJNR Am J Neuroradiol, 39:1776-1784. 